Arcos P., O. (2014). Plusvalías urbanas e impuesto predial, como fuente de financiamiento en la transformación de asentamientos precarios. Revista Lebret (6), Bucaramanga, Colombia: Universidad Santo Tomás, pp. 207-232 . ISSN 2145-5996.

\title{
Plusvalías urbanas e impuesto predial, como fuente de financiamiento en la transformación de asentamientos precarios*
}

\section{Urban surplus value and property taxes, as a source of financing in the transformation of slums}

Oscar Arcos Palma

\begin{abstract}
Resumen
La transformación de asentamientos precarios en las ciudades requiere de recursos de financiación que muchas veces exceden los presupuestos locales. En este artículo se propone como mecanismo de financiación la utilización de las plusvalías urbanas y de una porción de las rentas de la ciudad obtenibles en impuestos prediales, estos últimos como mecanismo temporal para apalancar dinámicas económicas en áreas con alto potencial económico. Los recursos así obtenidos, que se generan por los usos del suelo, son a su vez mecanismos de transformación urbana en lugares con asentamientos precarios y mejor aprovechamiento del suelo, en una perspectiva de dinámicas económicas y mejoramiento del bienestar.
\end{abstract}

\section{Palabras clave}

Renta del suelo, plusvalía, impuesto predial, financiación.

\section{Clasificación JEL: A10, B40, I38, R14}

\begin{abstract}
The transformation of slums in cities requires funding resources that often exceed local budgets. This paper proposes as a funding mechanism utilizing urban surplus value and rents obtainable city property taxes, the latter as a temporary mechanism to leverage economic dynamics in areas with high economic potential. The funds thus obtained, which are generated by land uses are themselves mechanisms for urban transformation in areas with slums and better land use, in a perspective of economic dynamics and improved welfare.
\end{abstract}

\section{Keywords}

Ground rent, surplus value, property taxes, financing.

* Este artículo hace parte de la investigación doctoral que el autor desarrolla: Renta del suelo urbano y bienestar Pertinencia del Teorema de GHV, de Vickrey (1977). Se inscribe, a su vez, en el proyecto de investigación sobre vivienda y hábitat que se adelanta en la línea de investigación de Economía Social, de la Facultad de Economía de la Universidad Santo Tomás, Seccional Bogotá.

1 Economista. Candidato a doctor en Ciencias Económicas, Universidad Nacional de Colombia. Docente de la Maestría en Ciencias Económicas en la Universidad Santo Tomás. Catedrático en la Maestría en Gobierno y Políticas Públicas de la Universidad Externado de Colombia. 


\section{Introducción}

En su artículo "The city as a firm", Vickrey (1977) propone el Teorema de GHV, basado en los aportes teóricos de George (1879) y Hotelling (1938). George, propuso un impuesto único sobre la renta del suelo diferencial, como instrumento de distribución de la riqueza; Hotelling, sugirió que un impuesto al valor de la localización de la tierra podría utilizarse para pagar los costos fijos de las plantas de energía eléctrica, las obras hidráulicas, los ferrocarriles y otras industrias, cuyos costos fijos son grandes, para bajar al nivel del costo marginal los precios cobrados por los servicios y productos de estas industrias. Vickrey señaló que las rentas del suelo en las ciudades podrían equipararse al monto de los subsidios requeridos para que las actividades con economías de escala vendan sus productos a precios equivalentes a sus costos marginales.

Este trabajo acoge la idea Vickrey, acerca de las rentas del suelo como equivalentes a subsidios requeridos para financiar bienes y servicios públicos, pero en este caso se hace una aproximación a la necesidad de transformar asentamientos precarios en las ciudades. Para dar fuerza argumental a la transferencia de recursos de rentas del suelo, a la transformación de asentamientos precarios, se tienen en cuenta los principios de la economía del bienestar, cuyos contenidos permiten valorar las medidas de política que puede tomar una administración pública local ${ }^{2}$ frente al problema de los asentamientos precarios. En efecto, el Óptimo de Pareto puede considerarse como un criterio para valorar acciones de una administración pública: indica, desde la perspectiva de la política pública, que cualquier medida que tome una administración local puede ser considerada deseable, si con ella se obtiene los siguientes efectos:

- Que todos los individuos de la ciudad se encuentren en una mejor situación.

- Que la situación de algún individuo en particular mejore, sin que empeore la situación de los demás.

Con respecto al problema de los asentamientos precarios, son dos los criterios que se anteponen a la aplicación de los principios del bienestar ${ }^{3}$ :

- La existencia de los asentamientos precarios obedece a fallas de mercado.

- Una actuación positiva promovida por la administración pública puede mejorar la situación de los asentamientos precarios ${ }^{4}$.

2 La mayor cuota de responsabilidad en la generación de suelo y en la solución de los problemas del hábitat (vivienda y entorno) corresponde a las administraciones locales.

3 Davis y Whinston (1961) hacen aportes de interés al incluir los elementos de la economía del bienestar en la perspectiva de los problemas de renovación urbana. He acogido parte de la argumentación de estos autores.

4 Al término mejorar le damos un alcance que se diferencia de acciones remediales tradicionales en la política pública, por ejemplo, ciertas acciones de mejoras de vivienda en asentamientos precarios, sin tener en cuenta las condiciones del entorno. 
Las reflexiones sobre las nociones de bienestar encuentran apoyo en el instrumental de Samuelson (1947), referido al "locus de posibilidades" de una comunidad. La comunidad se compone de dos individuos que actúan en el mercado. Para nuestro propósito, se utiliza el término ciudad a cambio de comunidad, y los dos individuos que conforman la comunidad son reemplazados por dos situaciones: la ciudad sin asentamientos precarios y la ciudad con asentamientos precarios, cuyas definiciones presentamos en el recuadro $1^{5}$. Para el desarrollo de los contenidos del presente trabajo tenemos en cuenta las siguientes denominaciones:

- Ciudad sin asentamientos: la denotamos como f

- Ciudad con asentamientos precarios: la denotamos como $\mathrm{p}$

Según el principio de bienestar referido a "una mejora en la situación de algún individuo en particular, sin que empeore la situación de los demás", los desplazamientos de las dos situaciones de la ciudad ( $f$ y $p$ ) en el "locus de posibilidades" de Samuelson (figura 1), establecen las condiciones del óptimo de Pareto. Este se puede lograr mediante impuestos y subsidios de suma fija. En términos simples, estas ideas significarían que las rentas que la ciudad genera en las áreas donde existe potencial económico $(f)$, y que las captura la administración pública, pueden subsidiar los costos de transformar asentamientos precarios en las ciudades.

Figura 1. Locus de posibilidades

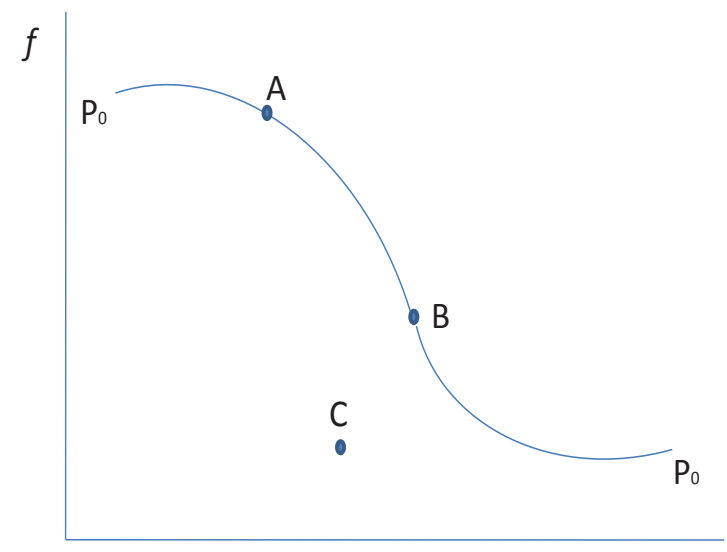

Fuente: Graaff, 1949.

$5 \quad$ Para una ampliación de los referentes conceptuales véase Arcos, O (2013). Nociones del bienestar en las interrelaciones ciudad sin asentamientos precarios- ciudad con asentamientos precarios Revista Lebret (5) Bucaramanga, Colombia: Universidad Santo Tomás, pp. 53-68 
Definiciones:

Ciudad con asentamientos precarios. Se refiere a áreas de la ciudad deteriorada o áreas que tuvieron origen ilegal o informal.

Ciudad sin asentamientos precarios. En oposición a lo anterior:

- Áreas consolidadas de la ciudad. Se asimila a las áreas con nivel de máxima rentabilidad alcanzada por las actividades económicas allí desarrolladas. Las categorías centros y subcentros de la ciudad son pertinentes a esta noción.

- Áreas de la ciudad con potencial económico. Áreas de la ciudad susceptibles de transformación -en virtud de su localización o valor de sitio-, mediante cambios de uso o escalamiento en intensidades de uso del suelo, a partir de procesos de planificación de la ciudad y de acciones de la administración pública. En esta categoría se incluyen: i) las áreas de la ciudad que antes fueron informales y que se fueron formalizando (legalizando) a través del tiempo y que se constituyen en escenarios con potencial económico; y ii) las áreas objeto de renovación urbana, a causa del deterioro progresivo.

Hemos señalado que la ciudad la componen $f$ y $p$. Tanto en $f$ como en $p$ se expresan escalas de preferencias definidas de los ciudadanos. Así, $f$ y $p$ representan índices de utilidad ordinal. Acogemos la convención de la economía del bienestar, de que $f$ aumenta su utilidad siempre que esa parte de la ciudad satisfaga en mayor medida sus preferencias. Cuando esto ocurre, su bienestar aumenta. Lo mismo es aplicable a $p$. En las interrelaciones que se presentan en la ciudad entre $f$ y $p$, se asumen economías y deseconomías externas.

Se pueden fijar, en un momento determinado, el nivel de la ciudad sin asentamientos precarios en $f_{0}$ y el nivel de la ciudad con asentamientos precarios en $p_{0}$. El nivel que podrá alcanzar el valor de la ciudad con asentamientos precarios $(p)$, dependerá de:

(1) El valor de la ciudad sin asentamientos precarios, $f_{0}$

(2) La oferta de bienes y servicios de que disponga la ciudad 6 .

(3) La posibilidad de:

6 Se considera la oferta existente y la oferta que puede suscitarse de la dinamización de actividades económicas en áreas de la ciudad con potencial económico. Tanto el potencial económico como las actividades que se puedan desarrollar en ciertas áreas de la ciudad, depende del nivel y la naturaleza de las relaciones de intercambio alcanzados por la ciudad internamente, con las regiones del país y con el resto del mundo. Los cambios en los usos del suelo y las actividades que se desarrollen en ellos dan señales del tipo de transformación que se da en bienes y servicios en ciertas áreas de la ciudad. 
Transformar bienes y servicios de una clase en bienes y servicios de otra, mediante el desarrollo de áreas de la ciudad con potencial económico.

Transformar asentamientos precarios, mediante la transferencia de rentas del suelo urbano, desde las áreas con potencial económico desarrolladas.

La magnitud de las transformaciones a las que se refiere el punto 3 depende de la cantidad de recursos de capital -públicos y privados- que se movilicen en ese proceso7. Al considerar las posibilidades de transformación, colocar a $p$ en la mejor posición posible, sujeto a $f=f_{0}$, se convierte en el problema de establecer las condiciones del óptimo general paretiano.

Suponemos la solución que dará el valor particular de $p$, como $p_{0}$, correspondiente a $f_{0}$. Al fijar $f$ en cualquier otro nivel se obtiene otro valor de $p$. Este procedimiento permite trazar un locus en el plano $(f, p)$. Este es el locus de posibilidades de Samuelson, que se ilustra con $\mathrm{P}_{0} \mathrm{P}_{0}$ en la figura 1. La ciudad con asentamientos precarios $p$, solo podrá mejorar a expensas de la ciudad sin asentamientos precarios $f$.

Con base en Samuelson y Graaff (1949) es posible afirmar que se aseguran los movimientos hacia el locus cuando se establecen las condiciones del óptimo general paretiano; los movimientos a lo largo del locus se logran mediante impuestos y subsidios de suma fija, que supone no cuestan nada recaudar y distribuir. Cualquier punto en el locus $\mathrm{P}_{0} \mathrm{P}_{0}$, siempre será mejor -en el sentido en que es mejor la posición de $f$ y de $p$-a cualquier punto en la parte inferior del locus ${ }^{8}$. Al respecto, apoyándonos en Samuelson, no podemos decir que un punto del locus, como A, sea mejor que un punto fuera del locus, como C, porque en A, la situación de los asentamientos precarios $(p)$ está peor que en C. Pero podemos afirmar que A es potencialmente mejor que $\mathrm{C}$, porque, necesariamente, existe un punto $\mathrm{B}$, en donde tanto $f$ como $p$ están mejor que en $\mathrm{C}$, y al que puede llegarse mediante una simple redistribución de la riqueza.

Señalamos también que una administración tiene por objetivo, entre otros asuntos relacionados con la eficiencia económica y el bienestar social, transformar los asentamientos precarios que han surgido en las ciudades, y para ello las normas han establecido un conjunto de instrumentos que las administraciones han venido aplicando como una forma de obtener dichas rentas. En este trabajo tenemos en cuenta dos instrumentos de captura de rentas del suelo: la plusvalía urbana y el impuesto predial, entendidos como proxy de rentas urbanas derivadas de las actividades económicas que se desarrollan en el suelo urbano de acuerdo con los usos residencial, industrial y comercial.

7 La aplicación de conocimientos de teoría dinámica del bienestar puede contribuir a las estimaciones de los recursos de capital requeridos para las transformaciones en la ciudad, de manera gradual y progresiva en el tiempo, dado que la ciudad es dinámica y cambiante en el tiempo.

8 Por los principios del Óptimo de Pareto, entre los puntos del locus no se puede juzgar, sin tener un criterio para las comparaciones interpersonales de bienestar. 


\section{Un par de consideraciones previas: Participación de las ciudades en el PIB nacional y coordinación administrativa}

El criterio de bienestar adoptado y el instrumental de Samuelson indican posibilidades para que una administración instaure reglas de juego a su favor, frente al reto de los asentamientos precarios, estableciendo una estructura de rentas apropiada desde la dinámica de nuevos usos del suelo o de uso intensivo en áreas con potencial económico en la ciudad. El hecho de basar el modelo en tales dinámicas presupone en las ciudades una importante actividad económica y una significativa participación en el PIB nacional. Las variables de productividad y competitividad en las ciudades constituyen referentes sobre su capacidad de generar valor agregado. Estos datos son importantes, en tanto prefiguran el margen de maniobra que tienen las ciudades para emprender transformaciones en sus territorios.

El modelo de generar y capturar rentas a través de la actuación de la administración, y de transferirlas a la transformación de los asentamientos precarios, presupone que la ciudad se ha persuadido de aceptar el esquema de redistribución que se propone, con el argumento de que la subsiguiente expansión de la renta en las áreas de la ciudad donde se transforman los asentamientos precarios tendrá efectos positivos en la ciudad, en su conjunto, en virtud de las interrelaciones que se establecen entre las distintas áreas de la ciudad (Richardson, 1968, p. 170).

Otro aspecto clave que se vislumbra como una condición necesaria y suficiente es la coordinación de la administración. Puesto que el objetivo de la política de transformación de asentamientos precarios exige de parte de la administración dinamizar y capturar las rentas del suelo urbano9, supone una coordinación de las decisiones de los agentes tanto en la dinámica que se genere en las áreas con potencial económico como en las áreas de asentamientos precarios. La coordinación previa deberá facilitar la concurrencia de los agentes y la puesta en marcha de las inversiones requeridas.

9 Smolka y Amborski (2000, p. 3) subrayan el papel que desempeña una administración pública en la generación de rentas: "En este sentido, aunque ciertas acciones tomadas directamente por los dueños privados de tierra pueden elevar el valor de la tierra, esta situación es más bien excepcional. La situación más frecuente es que esto último proviene de acciones realizadas por otros actores y no por los dueños privados de tierra, en particular, por las acciones del sector público, por ejemplo en los casos en que otorga permisos para el desarrollo de usos específicos de la tierra o densidades, o a través de inversiones en infraestructura. El incremento del valor de la tierra también puede darse a través de mecanismos de mercado, como por ejemplo, un incremento de la población urbana. En cualquiera de estos casos, queda claro que los dueños de la propiedad no realizaron nada para elevar el valor de la tierra y que puede ser socialmente deseable que el sector público capture todo o parte del incremento del valor". 


\section{Consideraciones sobre la actuación de una administración en la generación y captura de rentas}

Casi todos los países tienen en sus marcos normativos un conjunto de posibilidades de actuación del sector público en materia de políticas de gestión del suelo y de programas de vivienda. El marco normativo actual en Colombia, cuyo referente más importante es la Ley 388 de 1997, de Ordenamiento Territorial, contiene un amplio instrumental que las ciudades colombianas han venido aplicando de manera progresiva en el desarrollo de sus políticas de gestión del suelo, ordenamiento del territorio y programas de vivienda.

Bogotá es una de las ciudades con mayor desarrollo en el marco reglamentario y en la aplicación de los instrumentos normativos. Pero hasta el momento, la ciudad no ha hecho un ejercicio de estimar cuál es la magnitud de los costos que la ciudad debería asumir para solucionar problemas de asentamientos precarios y tampoco cuáles serían los beneficios.

El propósito de solucionar problemas de asentamientos precarios con rentas del suelo urbano obtenibles en áreas de potencial económico en las ciudades, exige a una administración responder los siguientes interrogantes:

- ¿Qué tipo de rentas y en qué magnitudes se generarían en las áreas de potencial económico de la ciudad, a lo largo del tiempo?

- ¿Qué rentas futuras se generarían en las áreas con asentamientos precarios susceptibles de transformación?

Sin ser exhaustivos, el procedimiento mínimo de las acciones de una administración implica:

- Tener en cuenta, como punto de partida: el nivel dado de imposición fiscal sobre la propiedad de los inmuebles, por tipo de uso del suelo, tanto en áreas con potencial económico como en las áreas con asentamientos precarios ${ }^{10}$.

- Movilización de fondos para dinamizar áreas con alto potencial económico (es una condición previa a la dinamización de la transformación de asentamientos precarios).

- La dinamización incluye la disposición de suelo para el desarrollo de los proyectos. La administración de una ciudad puede acudir a las figuras previstas en su corpus normativo. Se mencionan algunas:

10 Es de suponer que en los casos de ilegalidad no existe ningún tipo de imposición. 
- Asociación entre partícipes.

- Expropiación por vía administrativa. Este proceso evita, en teoría, procesos especulativos de alza de precios. Permite superar el obstáculo de propietarios renuentes a las iniciativas. Genera externalidades positivas.

- Adecuación de suelo, infraestructura (inversión pública).

- Desarrollo de los proyectos (la administración puede participar aportando suelo, como proporción de los recursos de capital y, por tanto, obtener de su aporte la proporción de beneficios correspondientes a la tasa de interés del mercado).

\section{Modalidades de captura de rentas}

\section{Impuestos}

Smolka y Ambroski (2000, p. 5), señalan: “... con respecto a los impuestos a la propiedad en general, y al valor de la tierra en particular, se debe destacar desde el comienzo que cualquier impuesto sobre el valor de la tierra es una forma de captura de plusvalías en tanto y en cuanto, por definición, los valores de la tierra están constituidos por incrementos acumulados del valor de la tierra. Por otra parte, dado que un impuesto al valor de la tierra reduce el flujo de ingresos esperados a ser generados por un cierto uso de la tierra, y el efecto de capitalización del valor actual de la tierra que ello produce, pueden así mismo ser vistos como una forma de captura de plusvalías".

El impuesto predial o impuesto a la propiedad es un impuesto periódico. En la mayoría de los países, el impuesto hace referencia al suelo y las mejoras, es decir, al precio global del inmueble Jaramillo (2006, p. 46); Morales (2003, 2004); Smolka y Ambrosky (2000); Smolka y Furtado (2001), entre otros. El hecho de que el impuesto predial se refiera al precio global del inmueble, dificulta la noción de neutralidad del impuesto aplicado a la tierra, según lo han señalado George, Hotelling y Vickrey, en tanto se supone que al gravar la tierra no ocasiona pérdidas de bienestar, ni en la esfera del consumo ni en la esfera de la producción. González (2010, p. 7) subraya que en la lógica de George, el terrateniente no puede trasmitir el impuesto a la tierra -que es el elemento completamente inelástico con respecto al precio-al comprador, a través de un aumento del precio. En este sentido, agrega:

... el impuesto sería un tributo 'neutral', y ello quiere decir que el gravamen a la renta no distorsiona los procesos de mercado. La neutralidad del impuesto es un argumento a favor de su implementación. La teoría convencional siempre se ha mostrado partidaria de la neutralidad tributaria, y si los tributos a la tierra cumplen con esta condición, no habría ninguna razón para rechazarlos.

Esto es claro, si el impuesto se refiriera solamente al suelo y no incluyera la edificación. No es muy clara la neutralidad en el caso de la edificación, puesto que 
parte del costo del impuesto puede fácilmente trasladarse al consumidor final. Es más fácil ver el tema de la neutralidad en los momentos en que se generan los incrementos del precio del suelo por acciones administrativas, en particular cuando se aplica, en el caso colombiano, la participación en plusvalía: el mecanismo de medición permite sustraer estrictamente, en las estimaciones, las variaciones de los precios del suelo, a través del denominado método residual Borrero $(1997,1998)$.

En teoría, un cálculo de las rentas del suelo a partir del impuesto predial es plausible. Habría que descontar la porción del gravado que correspondería a la edificación y su nivel de depreciación. No obstante, dado que la estimación del impuesto remite al criterio de área, y que el impuesto hace la distinción para distintos usos (residencial, industrial, comercial, institucional), la diferenciación de las rentas por cada inmueble en cada una de esas modalidades de uso se torna muy compleja. No solo habría que diferenciar las proporciones del impuesto en suelo y edificación, sino también habría que hacerla por tipo de uso.

\section{Tasas}

"Las tasas impuestas a los propietarios de tierras que se benefician de algún tipo de inversión pública son la forma más reconocida de capturar el incremento al valor de la tierra" (Smolka y Ambroski, 2000, pp. 6-7). Casi todos los países tienen normas que les permiten capturar, mediante tasas, los incrementos del valor de la tierra ocasionados por inversiones públicas ${ }^{11}$. El criterio es que la obra, por lo general, aumenta el patrimonio de los propietarios de los predios beneficiados y que por lo tanto ellos deben aportar a la financiación de la obra. La lógica de funcionamiento del gravamen es: aportes monetarios de los terrenos aledaños beneficiados por la obra -proporcional a la distancia-, vs monto de la inversión.

\section{Instrumentos regulatorios}

Existe también, en muchos países, un conjunto de normas y mecanismos que permiten capturar parte de los incrementos de los precios de suelo. Mencionamos dos, para el caso colombiano.

(i) La participación en plusvalias.

La noción que sustenta este mecanismo es la misma de la Contribución de Valorización: las acciones estatales en la ciudad a menudo provocan incrementos muy cuantiosos en los precios de determinados terrenos, favoreciendo enormemente a sus propietarios; parece razonable que los beneficiarios pasivos

11 En Estados Unidos y Canadá la tasa de impacto/cargo por desarrollo es de larga tradición: se destina a pagar la totalidad o parte del costo del capital de infraestructura. Colombia es uno de los pocos países de América Latina que viene aplicando una figura similar, desde hace décadas, para financiar obras públicas, obteniendo los recursos de los beneficiarios directos de las obras: la Contribución por Valorización. 
de estas acciones colectivas, hagan partícipes a la comunidad, al menos en parte, de estos beneficios (Jaramillo, 2000, p. 6).

Frente a las acciones que puede tomar una administración, en el marco de procesos de planificación, v. gr., cambios en la reglamentación de usos o intensidades de uso del suelo,

... los efectos sobre los precios parecen ser más previsibles tanto en su monto, como en su rango espacial, que en general está delimitado... [la participación en plusvalía], es una herramienta muy valiosa con fines redistributivos, como soporte de la financiación municipal y como elemento de planificación urbana [...], puede asimilarse a un impuesto capitalizado (por una sola vez) (Jaramillo, 2000, pp. 6-8).

En otros términos, se asemeja a un impuesto de suma fija, cuyo costo de recaudo y distribución es nulo. La base de cálculo de la plusvalía es la diferencia que resulta de comparar el precio del suelo antes de la acción administrativa y después de ella.

(ii) La distribución equitativa de cargas (costos) y beneficios de la urbanización. El mecanismo busca que los propietarios del suelo y los urbanizadores contribuyan -en virtud del incremento de los precios del suelo-, mediante cesiones del suelo, a cubrir la totalidad o parte de los costos generales (vías, espacio público, obras de infraestructura) atribuibles al desarrollo de sus terrenos ${ }^{12}$.

\section{Selección de modalidades de captura de rentas, para el modelo de interacción entre ciudad con asentamientos precarios $(f)$ y ciudad sin asentamientos precarios $(p)$}

Acogemos la afirmación que hace Jaramillo (2003, p. 12) con respecto a las rentas urbanas: "Las diferentes rentas que aparecen en la ciudad se imbrican en su territorio, de tal manera que cada lote individual soporta una combinación de ellas, que conforman lo que pudiéramos llamar la renta urbana total de cada uno". Esto significa que cualquiera de las modalidades que se han mencionado puede contener particularidades de las distintas rentas que se generan en suelo urbano.

El impuesto predial puede considerarse como un proxy de las diferentes rentas periódicas del suelo que aparecen en la ciudad, no obstante, sus limitaciones en cuanto a que no expresa la magnitud real de las rentas que se generan ${ }^{13}$. La participación en plusvalía refleja también la combinación de distintos tipos de rentas, pero a

12 En Bogotá, Colombia, el Decreto 252 de 2007 permite que el desarrollo de los proyectos incluyan un porcentaje del área a vivienda de interés social. No obstante, la obligación no parece un instrumento de captura de los incrementos en los precios del suelo, sino de compromisos relacionados con la disminución de la segregación y la solución a la no disponibilidad en la ciudad de suelo con destino a vivienda de interés social.

13 En el mejor de los casos, el impuesto predial se estima en promedio, en casi todos los países de América Latina, sobre el $70 \%$ del valor comercial de los inmuebles. 
diferencia de la fracción anual del precio del suelo que pueda verse reflejada en el impuesto predial, la participación en plusvalía refleja el precio del suelo en un momento de transacción comercial.

Para nuestros propósitos de diseñar un modelo de dinamización y generación de rentas urbanas en áreas de la ciudad con potencial económico y de transferencia para financiar la transformación de asentamientos precarios, proponemos el uso de dos modalidades de rentas del suelo: La participación en plusvalía y el impuesto predial. De este precisamos: la magnitud agregada del incremento en cada área con potencial económico desarrollada, y en cada área con asentamientos precarios transformada. Con esas dos fuentes de recursos, una administración puede aproximarse a una cuantificación de las rentas que se generan en determinadas áreas de la ciudad y en determinados periodos, y también puede establecer el margen de actuación, el ritmo y la intensidad de las inversiones que se requieran para transformar las áreas con asentamientos precarios. Las dos fuentes de recursos deben considerarse, durante el tiempo que se calcule puede tardar la administración en transformar los asentamientos precarios, como recursos con destinación específica.

Los argumentos para seleccionar estas dos modalidades de captura de rentas del suelo urbano con destino a la financiación de los asentamientos precarios, son la conveniencia y la viabilidad política. Proponer que una parte de la totalidad de las rentas del suelo se orienten a financiar la transformación de los asentamientos precarios puede ser convincente para la ciudad, atendiendo los principios de la economía del bienestar y sus implicaciones en el bienestar general. La participación en plusvalía es en Colombia un mecanismo que viene en proceso de consolidación, y las ciudades no tendrían objeción ante una propuesta de orientar la totalidad de los recursos que se generen por este concepto, a la solución de un problema que ha de contribuir al bienestar general. De otra parte, y en razón a que el impuesto predial financia otros servicios de la ciudad (educación, salud, obras de infraestructura...), es probable que al sugerir que sea solo la fracción correspondiente al incremento del impuesto predial en las áreas transformadas, y durante un periodo de tiempo determinado, la iniciativa no encuentre oposición.

No incluimos la Contribución por Valorización, bajo el criterio de que los recursos que se obtienen por este mecanismo se destinan a financiar el costo de las obras públicas. En este caso, no habría excedente para transferir a los proyectos de transformación de asentamientos precarios ${ }^{14}$. Tampoco consideramos alternativas de cambio en tasas impositivas vigentes en el sistema de impuesto predial.

14 Salvo si las obras públicas afectan directamente áreas de asentamientos precarios, podrían considerarse parte de sus costos como aportes de inversión de la administración en la transformación de dichas áreas. Otro tema es el derivado del Decreto 252 de 2007. Este Decreto obliga destinar, en los proyectos urbanos que se desarrollen en la ciudad, entre un $15 \%$ y un $20 \%$ del área útil a vivienda de interés social. La norma ha permitido que los empresarios urbanos trasladen la obligación, en determinadas circunstancias, a otras áreas de la ciudad. Esta posibilidad, encausada al objetivo de financiar la transformación de asentamientos precarios, puede apalancar recursos. El Decreto 252 de 2007 no es, en estricto sentido, un mecanismo de captura de rentas del suelo. 
Notamos con base en Jaramillo (1994)

$$
P S=\frac{R-\alpha}{i}
$$

Por tanto:

$$
\alpha=R-[(P S) *(i)]
$$

$$
\begin{aligned}
& P S=\text { Precio del suelo } \\
& R=\text { Renta } \\
& \alpha=\text { Impuesto } \\
& i=\text { Tasa de interés }
\end{aligned}
$$

Hemos señalado arriba, que asumimos el impuesto predial (impuesto a la propiedad: suelo y edificación), como la proporción de las rentas del suelo que captura de manera efectiva una administración, y, a su vez, tales impuestos son la expresión proxy del conjunto de rentas que se generan en un inmueble (figura 2).

Figura 2. Impuesto Predial y participación en plusvalía

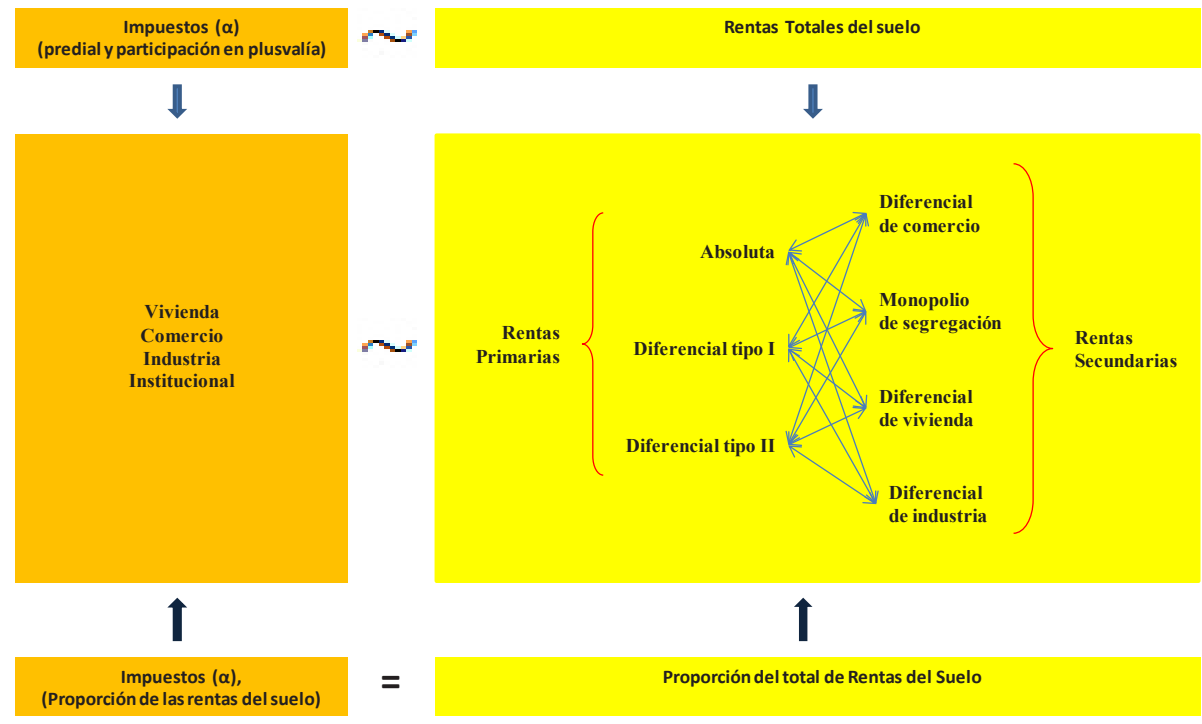

Fuente: autor. 
El impuesto podrá corresponder, en algún momento determinado del tiempo, a uno o a los dos impuestos que hemos seleccionado como fuentes de recursos: el impuesto predial -que se puede definir como un impuesto de propósito general-, y la participación en plusvalía -que se puede asemejar a un impuesto específico-:

$\alpha_{p r}=$ Impuesto predial

$\alpha_{p p}=$ Participación en plusbalía

\section{Balance de planificación para generar rentas en $f$ y transformar asentamientos precarios en $p$}

Consideramos pertinente, por tratarse de políticas públicas probables de una administración, el método de análisis coste-beneficio. Si el resultado esperado es mejorar las condiciones de bienestar del conjunto de la población en $f$ y $p$ en la ciudad, una aplicación coherente de los principios, el instrumental y el método de análisis a los que nos hemos referido significa tener en la mira el alcance de los impactos redistributivos. El método de análisis coste-beneficio por sí mismo, solo nos proporciona una idea de los resultados de los proyectos en forma individual. Pero en virtud de que el aspecto central de nuestra propuesta es la redistribución de las rentas del suelo -que se obtienen con el desarrollo de proyectos en áreas con potencial económico en la ciudad-, hacia proyectos orientados a resolver problemas de asentamientos precarios, significa que debe existir una relación entre las iniciativas que se tomen y desarrollen en $f$ y las iniciativas que se tomen y desarrollen en $p$. En otras palabras, nos adentramos en el terreno de los impactos de la redistribución. Al respecto, hacemos alusión al método coste-beneficio denominado Balance de Planificación $(\mathrm{BP})^{15}$, que nos permite prefigurar escenarios de la relación de los proyectos en $f$ y en $p$. Con base en el método BP, se establecen los siguientes tres criterios:

Criterio 1: Objetivo. En nuestro caso, el objetivo es dinamizar la generación de rentas del suelo urbano, a través del desarrollo de proyectos en áreas con potencial económico en la ciudad y redistribuirlas, a través de proyectos orientados a la transformación de asentamientos precarios.

Criterio 2: Identificación y clasificación.

Criterio 3: Distribución de costes y beneficios entre grupos específicos de población relevante.

15 La ventaja del BP es que permite “... reconocer la diversidad y los posibles conflictos de intereses y crea una base para el 'arbitraje' político, en las decisiones referidas a la planificación regional y urbana. Por lo tanto, la viabilidad de un proyecto es el resultado, en parte, de un proceso de negociación entre los inversores (que maximizan los rendimientos), los planificadores regionales, los gobiernos (¿maximizan los excedentes fiscales?) y los diversos grupos de la comunidad" (Richardson, 1986, p. 155). 
Para nuestra propuesta, añadimos tres criterios más:

Criterio 4: Simultaneidad.

Criterio 5: Gradualidad.

Criterio 6: Progresividad.

El método BP permite observar costes y beneficios en los proyectos que se desarrollen tanto en $f$ como en $p$. La articulación de las dinámicas de los proyectos en uno y otro lado, en términos de sus costos y sus beneficios, se orienta a dar consistencia al criterio del bienestar adoptado. En ella subyace la idea de que la pobreza y la miseria de la ciudad, particularmente la referida a los asentamientos precarios, depende esencialmente de la distribución de la renta. Las rentas del suelo urbano constituyen parte de la solución y, en nuestro caso, hemos especificado cuáles rentas deben contribuir a esa solución: las porciones correspondientes a los incrementos de las rentas periódicas que se reflejan en los incrementos del impuesto predial y la participación en plusvalía. Examinaremos los criterios 2 a 6 en las siguientes secciones.

\section{Áreas con potencial económico en $\boldsymbol{f}$ y áreas con asentamientos precarios en $p$ (criterio 2)}

La identificación de áreas con potencial económico debe responder a los interrogantes: ¿por qué desarrollar determinadas áreas?, ¿dónde?, ¿qué efectos se esperarían en torno al propósito de erradicar asentamientos precarios $\mathrm{y}$, en general, sobre la estructura espacial de la ciudad?

La identificación de áreas con potencial económico depende de las características particulares de cada ciudad. Un criterio básico y de común aceptación es la cercanía a centros de actividades con umbrales muy superiores a los normales. Las áreas con potencial económico cercanas a dichos centros atraerán, posteriormente, más actividades residenciales y no residenciales, puestos de trabajo y población. El efecto en la estructura espacial de la ciudad se expresa en cambios en los gradientes normales de las rentas y la densidad (Richardson, 1986, p. 240) ${ }^{16}$.

Se supone que, en virtud del potencial que tienen tales áreas -en contraste con las áreas que han alcanzado umbrales superiores-, y por los efectos de interdependencia locacional, tienden a generar procesos acumulativos (costes de distribución y de congestión bajos, y margen para el crecimiento de economías de aglomeración).

16 Richardson incluye dentro los umbrales, los complejos educativos, médicos o de oficinas, centros comerciales, terminales de transporte, entre otros. 
El método clásico de análisis de potencial de mercado lo propuso Harris $(1954)^{17}$. Fujita ([1999] 2000, pp. 41-42) señala:

La función típica del potencial de mercado mide el potencial de un determinado emplazamiento $r$ como la suma ponderada del poder adquisitivo de los demás emplazamientos $s$, siendo los coeficientes de ponderación una función decreciente de la distancia. De esta forma, una versión ampliamente utilizada pondera el poder adquisitivo inversamente a la distancia, con lo que el potencial de mercado en $r$ es:

$$
M_{r}=K \sum_{S} P_{S} / D_{r s}
$$

Donde:

$M_{r}=(r=1, \ldots, n)=$ Áreas con potencial de mercado

$K=$ Es una constante de proporcionalidad que depende de la unidad de medida adoptada

$D_{r s}=$ Distancia de $r$ a $s ;(s=1, \ldots, n)$

$P_{s}=$ Poder adquisitivo en $s$

Señalamos, para el conjunto de la ciudad (figura 3):

f: $\quad$ Ciudad sin asentamientos precarios

$f_{s}: \quad$ Ciudad sin asentamientos precarios, con umbral superior de acti vidad

$f M_{r}$ : Ciudad sin asentamientos precarios, con potencial económico. Incluye las posibilidades de rehabilitación y renovación urbana.

17 Camagni (2005, p. 83) señala: "El concepto de potencial económico espacial desciende de una analogía con la física gravitatoria. Así, se afirma que todo cuerpo de masa unitaria $a$, situado en el campo gravitatorio de una masa $b$, posee una energía potencial igual al trabajo que $a$ haría al caer sobre $b$ :

$$
E_{a b}=K M_{b} / \delta_{a b}
$$

La variable $\delta$, expresa la distancia. Más en general, suponiendo diversos campos de fuerzas, el potencial total producido sobre a por un conjunto de masas $M_{j}(j=1 . . n)$, se define como:

$$
E_{a}=K \sum_{j} M_{j} \delta_{a j}
$$

La aplicación de esta ecuación a los fenómenos económicos y espaciales es fácil y directa, con un grado de libertad añadido dado por el exponente $\gamma$, de la variable que expresa la distancia $(\delta)$, que puede ser diferente de 1 , para asi tener en cuenta la distinta fricción espacial que tiene lugar en los distintos tipos de fenómenos:

$$
E_{a}=K \sum_{j} M_{j} \delta_{a j}^{y}
$$

La interpretación económica más general del concepto de potencial está relacionada con [...] un concepto de accesibilidad o interacción generalizada: una característica que proviene de la posición relativa de un lugar en el interior de un espacio geográfico en el cual están localizadas n masas con el cual dicho lugar entre en una relación de interacción. [...] el concepto puede ser considerado como indicador de 'flujos potenciales' y como un indicador de "posición [...] llevando al extremo la analogía con la física clásica, podríamos definir el potencial económico-espacial como energía potencial de localización". 


\section{p: $\quad$ Ciudad con asentamientos precarios}

px: Ciudad con asentamientos precarios, sin solución (vulnerables por riesgos ambientales no mitigables)

pM: Ciudad con asentamientos precarios, con algún nivel de potencial económico para transformación

A partir de (3) se identifica la totalidad de las áreas $M_{r}$ permite una escala de valoración que va desde la situación más crítica en áreas con asentamientos precarios $(-n)$, susceptibles de transformación, hasta las áreas con mayor potencial económico en la ciudad ( $n$ ) (figura 3). La línea que dentro del conjunto $M_{r}$ separa las áreas en $f \mathrm{y}$ en $p$, se establece a partir de las características que definen los asentamientos precarios. Asumimos que, aún en situación de ilegalidad, la tierra que ocupan dichos asentamientos tiene valor. Por tanto, la tierra en peor situación en los asentamientos precarios, genera o ha generado renta absoluta. Se excluyen de $M_{r}$ las áreas de la ciudad que, por su desarrollo, han alcanzado los umbrales más altos de actividad y también las áreas con asentamientos precarios en riesgo ambiental no mitigable.

Figura 3. Escala de clasificación de áreas

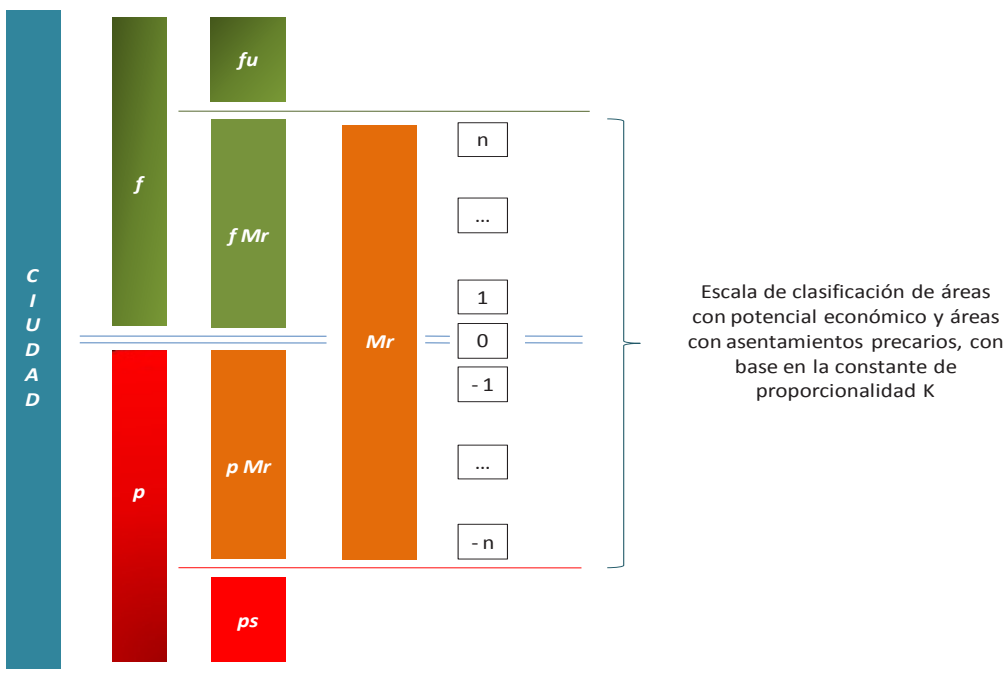

Fuente: autor. 


\section{Distribución de costes y beneficios entre grupos de población relevante (criterio 3)}

El método de análisis coste-beneficio ${ }^{18}$ permite identificar en un proyecto los costos de producción, incluidos en ellos los beneficios del capital estimados con una tasa media de ganancia en el mercado. Y, además, el valor residual que corresponde al valor de la renta del suelo. A este valor se le puede denominar beneficio neto y es la parte que representa la cantidad tota ${ }^{19}$ que la administración podría utilizar para mejorar la situación de los asentamientos precarios en $p$, bajo el supuesto de que la redistribución tiene costos nulos.

La estimación de los costes debe hacerse en cada uno de los proyectos que la administración decida promover para su desarrollo, tanto en las áreas con potencial económico en $f$, como en las áreas con asentamientos precarios en $p$, donde habrán de llevarse a cabo los procesos de transformaciones deseadas en la ciudad. Uno de los procedimientos sencillos y de uso común en el método de análisis costes-beneficios es el de la estimación del valor presente neto $(\mathrm{VPN})^{20}$. Se supone que cualquier proyecto con un VPN positivo, debe desarrollarse.

Los costes básicos son:

- Compra de tierra o inmuebles.

- Demoliciones.

- Infraestructura física de entorno: vías, espacio público y equipamientos.

- Infraestructura de redes de servicios públicos domiciliarios.

- Costes de producción del proyecto.

- Intereses de capital.

18 En la práctica, el método se utiliza para las operaciones urbanas. En las normas y los instrumentos colombianos, relacionados con el desarrollo urbano territorial se utiliza la terminología cargas y beneficios. Se asimila a costes-beneficios.

19 No sobra insistir en la aclaración de que en la práctica las administraciones no capturan la totalidad de las rentas del suelo. Por tanto mantenemos el criterio de que el impuesto predial y la participación en plusvalía corresponden a una proporción de la totalidad de las rentas.

20 El procedimiento se basa en estimar el beneficio neto, B, en cada periodo de tiempo, restando los costes de los beneficios:

$$
\begin{aligned}
B_{1}= & \left(b_{1}-c_{1}\right) \\
B_{2}= & \left(b_{2}-c_{2}\right) \\
& \cdots \\
& \cdots \\
B_{t}= & \left(b_{t}-c_{t}\right)
\end{aligned}
$$

$b_{1}=$ beneficios brutos en el periodo $1 . c_{1}=$ costes brutos en el periodo 1 . Teniendo en cuenta el tipo de interés, el VPN se estima así:

$$
\begin{array}{r}
\mathrm{VPN}=\mathrm{Bo}+\frac{B_{1}}{(1+r)}+\frac{B_{2}}{(1+r)^{2}}+\ldots+\frac{B_{n}}{(1+r)^{n}} \\
\text { donde r es la tasa de descuento, o VPN }=\sum_{t=0}^{t=n} \frac{B_{t}}{(1+r)^{t}}
\end{array}
$$


- Costes de reubicación de población.

Los beneficios generales son:

- Rentas del suelo o recursos fiscales que gravan la propiedad urbana ${ }^{21}$.

- Rentabilidad del capital o tasa de ganancia media para los promotores, empresarios inversionistas, propietarios del suelo y de inmuebles.

Los grupos de población relevante son comunes en los proyectos por desarrollar en $f \mathrm{y}$ en $p$ :

- Propietarios del suelo

- Promotores

- Productores/Operadores empresariales

- Residentes actuales y nuevos residentes/Población objetivo

En particular, la administración puede fungir en el rol de promotor/gestor y habilitador del suelo. Incluso, podrá convertirse en algunas circunstancias en propietario temporal del suelo ${ }^{22}$, haciendo uso de recursos normativos (v. gr., expropiaciones). Al participar en cualquiera de esos roles, la administración establece montos periódicos de inversiones de capital propio ${ }^{23}$, dependiendo de los proyectos, los cuales constituyen su aporte al conjunto de las inversiones totales requeridas. Dependiendo también de la visión que se haya establecido en el mediano y el largo plazo, en cuanto a las transformaciones de la ciudad en $f$ y en $p$, mediante el mecanismo de balance de planificación, la administración podría considerar al menos dos alternativas de redistribución de las rentas del suelo generadas y obtenidas por la administración, así como los beneficios obtenidos por aportes de capital propio a los proyectos en $f$, así:

i) Redistribuir la totalidad de rentas y beneficios propios a los proyectos en $p$.

ii) Redistribuir una proporción de rentas y beneficios a nuevos proyectos en $f$ donde se prevea la obtención de rentas elevadas -bajo un criterio de acelerar el desarrollo de áreas generadoras de rentas altas-y una proporción a los proyectos en $p$.

21 David y Whinston (1961, p. 317) señalan al respecto que “... el valor actualizado del impuesto sobre la propiedad urbana repercutirá, probablemente, sobre el factor fijo, el suelo. Si el proyecto tiene éxito, las nuevas estructuras y edificaciones tendrán un valor más elevado que las antiguas y, por consiguiente se contabilizará una adición neta de los ingresos fiscales, adición que habrá que descontarse para obtener su valor actual y que deberá considerarse como un ingreso generado por el proyecto. De este modo, podemos establecer una comparación entre ingresos y gastos, y el proyecto vendrá justificado tan solo cuando aquellos superen a estos".

22 Lo ideal sería una aproximación a la condición de propietario permanente del suelo. Esta apreciación se asemeja a la señalada por Walras (Alfonso, 2011, p. 8): “... la propiedad colectiva de la tierra, [...], satisface las condiciones de equidad al mismo tiempo que las condiciones igualitarias en materia de repartición de la riqueza [...]".

23 Además de los recursos propios de las ciudades se incluyen en ellos las subvenciones del nivel nacional y los créditos internos y externos. 


\section{Simultaneidad (criterio 4)}

El modelo presupone un desarrollo simultáneo de proyectos en $f$ y en $p$. Con este criterio se pretende que la interacción entre $f$ y $p$ produzca los resultados esperados según los principios de la economía del bienestar. Como se busca que las rentas del suelo generadas y obtenidas por la administración en f se redistribuyan en proyectos orientados a solucionar problemas de asentamientos precarios en $\mathrm{p}$, debe minimizarse la incertidumbre asociada a los flujos futuros de beneficios y costes. Esto significa que debe adoptarse un periodo de inversión idéntico para el proyecto que se desarrolle en $f$ y para el proyecto que se desarrolle en $p$. Significa, además, que la administración establece como base una igualdad entre el monto de las rentas obtenibles en el (los) proyecto (s) en $f$ con el monto de las inversiones que transfiere a $p$, de manera que exista una aproximación a la igualdad entre los beneficios generales obtenidos y los costes generales de erradicar asentamientos precarios. La noción del "locus de eficiencia" es pertinente.

\section{Gradualidad (criterio 5)}

El criterio 2 permite establecer diferentes escenarios alternativos de desarrollo de las transformaciones deseadas en $f \mathrm{y}$ en $p$. La clasificación del conjunto de áreas contenidas en en la escala de valoración entre $(-n)$ y $(n)$ (figura 3$)$, permitiría a su vez establecer grados de complejidad de los proyectos probables. En aras de la sencillez, asociamos el nivel de complejidad al nivel de costos de los proyectos. Con esta idea, es de suponer que los proyectos más costosos en transformación de asentamientos precarios en $p$ corresponden a los grupos poblacionales en condiciones más críticas de pobreza, o cuyas condiciones geográficas elevan los costos de la provisión de infraestructuras. Y, de otra parte, los costos de los proyectos en $f$ pueden asociarse, por simplicidad, al tamaño de los proyectos y al valor de sitio o de posición.

Dependiendo de las capacidades económicas de la ciudad, de los escenarios que la ciudad se plantee frente al "locus de posibilidades" y de sus prioridades, la administración podrá establecer distintos escenarios de actuación. Mencionamos dos, para ilustrar ${ }^{24}$ :

- Desarrollar áreas en $f M_{r}$ más cercanas al nivel $(n)$ de valoración y transferir las rentas a $p M_{r}$ a los niveles más críticos de asentamientos precarios, cercanos a $(-n)$.

- Desarrollar áreas en $f M_{r}$ más cercanas al nivel $(n)$ de valoración y transferir las rentas a $p M_{r}$ a los niveles menos críticos de los asentamientos precarios, más alejados de $(-n)$.

24 El espectro de decisiones que tomaría una administración se parecen al espectro de decisiones que se tomarían para superar niveles de pobreza. Los dos escenarios que se mencionan se parecen a las opciones que tiene una administración de privilegiar a quienes se encuentran más cerca de la línea de pobreza o a los más alejados de ella. 


\section{Progresividad (criterio 6)}

Una ciudad no puede resolver el tema de los asentamientos precarios en corto plazo. Se suponen, bajo el esquema propuesto, desarrollos progresivos en el largo plazo $^{25}$.

\section{Generación de rentas en el conjunto de áreas en $f$ y $p\left(M_{r}\right)$}

En un momento determinado del tiempo $\left(t_{o}\right)$, la ciudad puede presentar cuatro situaciones generales (figura 4):

- Áreas con umbrales altos de actividad, donde las rentas y los precios del suelo han adquirido los máximos niveles $\left(f_{u}\right)$. En las ciudades, corresponden a los centros o subcentros. En la curva AA, el punto $x_{1}$ indica el máximo valor alcanzado en $t_{o}$.

- Áreas con potencial económico que incluyen la opción de rehabilitación urbana $\left(f M_{r}\right)$. En $\mathrm{AB}$, el punto $y_{1}$ es el máximo en rentas y precios del suelo en $t_{o}$.

- Áreas con potencial económico que incluyen la opción de renovación urbana $\left(f M_{r}\right)$. En AC, el máximo es $z_{1}$.

- Áreas con asentamientos precarios con posibilidades de transformación $\left(p M_{r}\right)$. En AD el máximo es $w_{1}$.

Figura 4. Situaciones generales

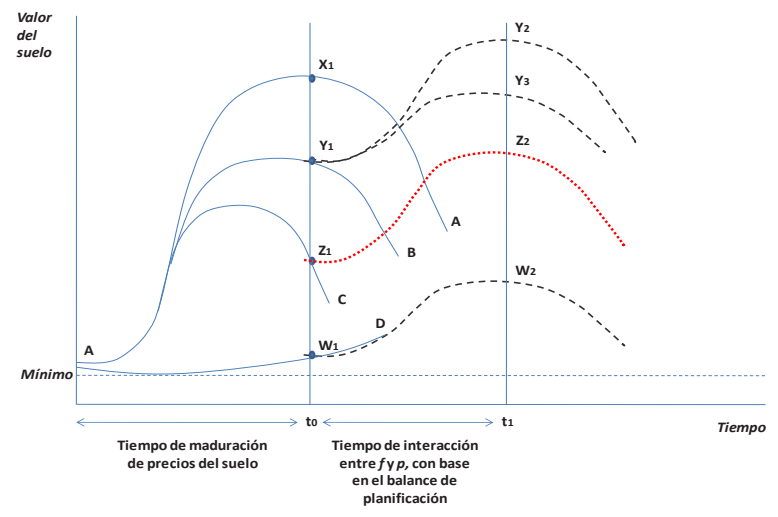

Fuente: autor, con base en Borrero O. (1998).

25 Según la magnitud de las áreas con asentamientos precarios y áreas susceptibles de rehabilitación y renovación urbana, una ciudad tendría al menos 50 años de actividades para lograrlo. Según el DNP, Bogotá tenía 1800 hectáreas con asentamientos precarios al finalizar la primera década de este siglo (el 15\% del área residencial total de la ciudad). La administración promovió el desarrollo de 300 hectáreas para vivienda de interés social en un tiempo promedio de 7 años. Suponiendo que la ciudad limita el incremento de asentamientos precarios, un ritmo de desarrollo, según el modelo propuesto, significaría un tiempo mínimo de 42 años. 
Retomando (2), $(\alpha=R-[(P S) *(i)])$, la situación en $t_{0}$ es:

$$
\begin{gathered}
\alpha_{0}=R_{0}-\left[\left(P S_{\mathrm{o}}\right) *\left(i_{0}\right)\right] \\
\alpha_{0}=\sum_{i=1}^{n} \alpha_{p r_{0}}
\end{gathered}
$$

$\alpha_{0}$ es la totalidad del impuesto predial $\left(\alpha_{p r_{0}}\right)$ de todos los inmuebles del conjunto de áreas identificadas en $M_{r}$, en el tiempo $t_{0}$, como proporción de las rentas del suelo en esas áreas.

Con acciones de rehabilitación y renovación urbana, la situación en $t_{0 \rightarrow 1}$, que se considera un periodo de transición mientras se desarrollan los proyectos, es:

$$
\alpha_{0 \rightarrow 1}=R_{0 \rightarrow 1}-\left[\left(P S_{0 \rightarrow 1}\right) *\left(i_{0 \rightarrow 1}\right)\right]
$$

Donde:

$$
\begin{aligned}
& P S_{0 \rightarrow 1}=\mathrm{PS}_{0}+\Delta \mathrm{PS}_{0} \\
& \alpha_{0 \rightarrow 1}=\sum_{i=1}^{(n+\Delta n)} \alpha_{0 \rightarrow 1} \\
& i=\text { inmuebles } \\
& \alpha_{0 \rightarrow 1}=\sum_{i=1}^{(n+\Delta n)}\left(\alpha_{p r_{0 \rightarrow 1}}, \alpha_{p p_{0 \rightarrow 1}}\right)
\end{aligned}
$$

$\alpha_{0 \rightarrow 1}$, incluye el impuesto predial $\left(\alpha_{p r_{0 \rightarrow 1}}\right)$ y la participación en plusvalía $\alpha_{p p_{0 \rightarrow 1}}$. 
Para los años sucesivos al periodo de transición $\mathrm{t}_{1 \rightarrow}$, se plantea la siguiente situación:

$$
\alpha_{1 \rightarrow}=R_{1 \rightarrow}-\left[\left(P S_{1 \rightarrow}\right) *\left(i_{1 \rightarrow}\right)\right]
$$

Donde:

$$
P S_{1 \rightarrow}=P S_{0 \rightarrow 1}+\Delta P S_{0 \rightarrow 1}
$$

Hemos señalado que, por viabilidad y conveniencia política, los recursos de las rentas del suelo destinadas a la transformación de los asentamientos precarios corresponderían a las rentas provenientes de la participación en plusvalía y al incremento del impuesto predial, una vez los proyectos se hayan desarrollado ${ }^{26}$. Así, las rentas destinadas a financiar asentamientos precarios -que denotamos $\alpha$ fap-, serían los recursos de participación en plusvalía en el periodo de transición $t_{0 \rightarrow 1}$, más la diferencia entre la nueva situación de los impuestos prediales en el periodo $t_{1}$ (después del desarrollo de los proyectos) y los impuestos prediales en $t_{0}$ (antes del desarrollo de los proyectos). Es evidente que, tratándose de proyectos de rehabilitación, que prefiguran densificación del suelo, y de mejor aprovechamientos del suelo en áreas con asentamientos precarios, donde se prevé también la densificación, el total del impuesto predial incluiría la ampliación de la base de tributación generada por la densificación.

$$
\alpha f a p=\alpha_{p p_{0 \rightarrow 1}}+\left(\alpha_{p r_{1}}-\alpha_{p r_{0}}\right)
$$

$\alpha$ fap, es el monto requerido para subsidiar costos de transformación de asentamientos precarios.

\section{El cambio de las participaciones en la estructura de rentas del suelo como método de evaluación ex post}

Para la evaluación ex post de las transformaciones en la ciudad, a partir de las dinámicas generadas en $f \mathrm{y}$ en $p$ con base en la aplicación del modelo propuesto,

26 La temporalidad de utilización de los incrementos podría establecerse de acuerdo con las magnitudes de recursos que la administración tendría que transferir a los proyectos con asentamientos precarios. El ejercicio de estimar costos y beneficios en cada uno de los proyectos por desarrollarse en el conjunto de áreas en $\left(f M_{r}\right)$ y $\left(p M_{r}\right)$ daría señales para establecer la temporalidad. 
creemos que el método de cambio de las participaciones, adaptado a nuestros propósitos, sería pertinente ${ }^{27}$. Permitiría medir el cambio propiciado por la aplicación del modelo, tanto en las áreas de $f$ como en las de $p$, con respecto al conjunto de la ciudad en un periodo de tiempo. En particular, se podrían estimar las magnitudes de crecimiento real en la estructura de rentas de la ciudad y, por tanto, de los recursos fiscales a partir de la gestión de la administración con la aplicación del modelo, en contraste con el crecimiento esperado en la ciudad siguiendo su tendencia histórica (sin aplicación del modelo).

El método incluye los siguientes pasos:

$$
\Delta \alpha_{p r} M_{r}=X \alpha_{p r} M_{r}+S \alpha_{p r} M_{r}
$$

El crecimiento de los impuestos prediales $\left(\alpha_{p r}\right)$ en $M_{r}$ es igual a su participación $(X)$ frente al total del impuesto predial de la ciudad $\left(\alpha_{t}=Z\right)$, más el cambio $(S)$ en su participación, suscitado por la aplicación del modelo. El método descompone $S$ en:

$S_{d}=$ Mide la tasa de crecimiento del impuesto predial en las diferentes áreas de $M_{r}$ en relación con el total del impuesto predial de la ciudad.

$S_{p}=$ Mide el efecto de la composición del impuesto predial en $M_{r}$, según tipo de impuesto (vivienda, comercio, industria, institucional).

Para simplificar la notación:

$\Delta \alpha_{p r} M_{r}=Y=$ Incremento del impuesto predial en las áreas $M_{r}$

$\alpha_{T}=Z=$ Impuesto predial total de la ciudad $(Z)$

$X \alpha_{p p} M_{r}=X=$ Participación del puesto predial en $M_{r}$ frente al total de la ciudad

$S \alpha_{p p} M_{r}=S=$ Cambio de la participación del puesto predial $(\mathrm{X})$

Por tanto:

27 Richardson (1986, p.156) expone las ventajas y limitaciones del método. En nuestro caso, dada la dificultad de estimar con precisión las rentas del suelo, la información del impuesto predial, por un lado, y la información de las rentas generales por desarrollo de actividades económicas en distintas áreas de la ciudad y su correspondiente contribución al PIB, daría cuenta de las transformaciones en los niveles de bienestar por áreas y grupos poblacionales, según lo previsto en las áreas de intervención a partir del balance de planificación. Se hace necesario, para la aplicación del método, establecer la situación inicial general de la ciudad, de manera que se puedan realizar comparaciones inter-temporales para evaluar los impactos de aplicación del modelo. 


$$
\begin{aligned}
& \mathrm{Y}=\mathrm{X}+\mathrm{S} \\
& \mathrm{S}=\mathrm{Y}-\mathrm{X} \\
& t=\text { Periodo final } \\
& 0=\text { Periodo inicial }
\end{aligned}
$$

Como X es la variación de los impuestos prediales si la tasa de crecimiento de los impuestos hubiese sido idéntica a la de la ciudad, el cambio total es igual a:

$$
\begin{aligned}
& S=\left(Y_{t}-Y_{0}\right)-\left(Y_{0}-\left[\frac{Z_{t}}{Z_{0}}\right]-Y_{0}\right) \\
& S=Y_{t}-\left(\frac{Z_{t}}{Z_{0}}\right) Y_{0}
\end{aligned}
$$

Descomponiendo S:

$$
S_{d}=\sum_{i}\left[Y_{t}^{i}-\left(\frac{z_{t}^{i}}{z_{0}^{i}}\right) Y_{0}^{i}\right]
$$

$i$ = impuesto predial según actividad económica sobre el suelo (industria, vivienda, comercio)

$$
\begin{aligned}
& S_{p}=Y_{t}-\left(\frac{Z_{t}}{Z_{0}}\right) Z_{0}-\sum_{i}\left[Y_{t}^{i}-\left(\frac{z_{t}^{i}}{z_{0}^{i}}\right) Y_{0}^{i}\right] \\
& S_{p}=\sum_{i}\left[\left(\frac{z_{t}^{i}}{z_{0}^{i}}\right)-\left(\frac{Z_{t}}{Z_{0}}\right)\right] Y_{0}^{i}
\end{aligned}
$$

Con la técnica presentada podrá describirse la estructura de impuestos prediales -como símil de rentas del suelo- en cada una de las áreas identificadas en, antes y después de las transformaciones realizadas mediante el desarrollo de proyectos de rehabilitación y renovación urbana y de transformación de asentamientos precarios. Así, una administración podrá evaluar los impactos generados en la estructura de recursos fiscales provenientes del uso del suelo urbano. 


\section{Conclusiones}

En los tiempos actuales se presentan retos a los gobiernos locales para resolver problemas relacionados con bienes y servicios en las ciudades. La reflexión en torno a postulados teóricos sobre las rentas del suelo urbano y la teoría del bienestar es pertinente. El elemento articulador es el subsidio. En el caso de la teoría del bienestar, el matiz que se le da a ciertas características del óptimo de Pareto se apoya en el criterio de las compensaciones. El símil subsidios - compensaciones permite articular los elementos teóricos de las rentas del suelo y del bienestar y, en sentido práctico, proponer alternativas para el financiamiento de bienes y servicios públicos o también de transformación de asentamientos precarios en las ciudades a través de políticas públicas de vivienda y hábitat.

\section{Referencias}

Alfonso, O. (2011). La cuestión de las plusvalías urbanas: viejas discusiones, nuevas perspectivas. Plusvalías Urbanas. Fundamentos Económicos y Jurídicos en Colombia. (pp. 27-58). Editorial Departamento de Publicaciones Universidad Externado de Colombia.

Arcos, O. (2013). Nociones del bienestar en las interrelaciones ciudad sin asentamientos precarios - ciudad con asentamientos precarios. Revista Lebret 5, Bucaramanga, Colombia: Universidad Santo Tomás. (pp. 53,-68)

Borrero, O. (1997). Valor del suelo urbano en Bogotá. Lonja de Propiedad Raíz de Bogotá, Bogotá.

Borrero, O. (1998). Avalúo y rentabilidad de inmuebles titularizados en Colombia. Colección OIKOS, t. 3. Bogotá.

Camagni, R. (2005). Economía urbana. Barcelona: Antoni Bosch (Ed.). (Versión original).

Davis, O., y Whinston, A. (1968). Aspectos económicos de la renovación urbana. En Análisis de las estructuras territoriales, (pp. 300-318). Recopilación de Bernardo Secchi. Barcelona: Gustavo Gili S.A.

Fujita, M., Krugman, P., and Venables, A. (septiembre, 2000). The spatial economy, Massachusetts Institute of Technology. (En español: Economía espacial: las ciudades, las regiones y el comercio internacional). Barcelona: Ariel Economía.

George, H. (1980). Progreso y miseria, Madrid: Editorial Prometeo.

González, J. I. (2010). El interés público y la participación en plusvalías. En Alfonso, O. (Ed.). La participación en plusvalías es un asunto de interés público. Bogotá: Universidad Externado de Colombia. 
Graaff, J. de V. (1949). Las estructuras óptimas de los aranceles. The Review of Economic Studies, 17, 47-59.

Hotelling, H. (1974). El bienestar general en relación con los problemas de tributación y de fijación de las tarifas de ferrocarriles y servicios públicos. Selección de Arrow, K. J., y Scitovsky, T. La economía del bienestar. México: Fondo de Cultura Económica.

Jaramillo, S. (1994). Hacia una teoría de la renta del suelo urbano. Bogotá: Ediciones Uniandes, Instituto Geográfico Agustín Codazzi.

Morales-Schechinger, C. (2003). La imposición a la propiedad en el financiamiento de las ciudades frente a la globalización. Mimeo. Cambridge, MA.: Lincoln Institute of Land Policy,

Richardson, H. W. (1986). Economía regional y urbana. Madrid: Alianza Editorial.

Samuelson, P. A. (1947). Foundations of Economic Analysis, Cap. VIII. Cambridge, Mass.

Smolka, M. (1981). Precio de la tierra y valorización inmobiliaria: esbozo para una conceptualización del problema. Revista Interamericana de Planificación, $X V(60), 70-89$. México, D.F.

Smolka, M., y Amborski, D. (2000). Apropiación de valor para el desarrollo urbano: una comparación Inter-Americana. Mimeo. Cambridge, MA.: Lincoln Institute of Land Policy.

Smolka, M., y Furtado, F. (2001). Plusvalías urbanas en Brasil: creación, recuperación y apropiación en la ciudad de Sao Paulo. En: Smolka, M. y Furtado, F. (Eds.). Recuperación de plusvalías urbanas en América Latina. Alternativas para el desarrollo urbano, (pp. 37-70). Cambridge, MA.: Eurelibros, Lincoln Institute of Land Policy, Pontifica Universidad Católica de Chile, Santiago de Chile.

Smolka, M. y Furtado, F. (julio, 2001). Lecciones aprendidas de la experiencia en América Latina con la recuperación de plusvalías. Land Lines, 4(13). Cambridge MA. LILP.

Smolka, M. y Furtado, F. (Ed.) (2001) Recuperación de plusvalias urbanas en América Latina. Alternativas para el desarrollo urbano. Cambridge, MA. Editorial Lincoln Institute of Land Policy, - Pontificia Universidad Católica de Chile. Santiago de Chile

Vickrey, W. (1977). The city as a firm. In Arnott, R., Arrow, K., Atkinson, A., Dreze, J. (1994) Selected Papers by William Vickrey Cambridge University Press, Cambridge, pp. 339-349. Ed. Public Economics. 